\title{
Advantage of Leakage of Essential Metabolites for Cells
}

\author{
Jumpei F. Yamagishi $\odot,{ }^{1}$ Nen Saito, ${ }^{2, *}$ and Kunihiko Kaneko ${ }^{1,3, \dagger}$ \\ ${ }^{1}$ Graduate School of Arts and Sciences, The University of Tokyo, 3-8-1 Komaba, Meguro-ku, Tokyo 153-8902, Japan \\ ${ }^{2}$ Graduate School of Science, The University of Tokyo, 7-3-1 Hongo, Bunkyo-ku, Tokyo 113-0033, Japan \\ ${ }^{3}$ Center for Complex Systems Biology, Universal Biology Institute, The University of Tokyo, \\ 3-8-1 Komaba, Meguro-ku, Tokyo 153-8902, Japan
}

(Received 20 May 2019; published 28 January 2020)

\begin{abstract}
Microbial cells generally leak various metabolites including those necessary to grow. Why cells secrete even essential chemicals so often is, however, still unclear. Based on analytical and numerical calculations, we show that if the intracellular metabolism includes multibody (e.g., catalytic) reactions, leakage of essential metabolites can promote the leaking cell's growth. This advantage is typical for most metabolic networks via "flux control" and "growth-dilution" mechanisms, as a general consequence of the balance between synthesis and growth-induced dilution with autocatalytic reactions. We further argue that this advantage may lead to a novel form of symbiosis among diverse cells.
\end{abstract}

DOI: 10.1103/PhysRevLett.124.048101

In microbial communities, diverse species or strains coexist rather than exist as a single fittest type competitively excluding all others, even when limited by a single nutrient $[1,2]$. Such coexistence is due to cell-cell interactions mediated by the intercellular exchange of leaked chemicals that can create new niches [2-4]. Even under isolation conditions, microbial cells secrete various metabolites, despite the naïve expectation that leakage and loss of metabolites will hinder cellular volume growth. Of course, it is evident that every cell should dispose of inhibitory or waste byproducts [5-8] or toxic compounds $[9,10]$. Recent studies on the exometabolome, however, revealed that many microorganisms leak (and take up) a variety of metabolites that are necessary for growth, including most intermediates of central metabolism $[11,12]$. Counterintuitively, the leakage of various metabolites is experimentally observed even when the supplied resource is scarce [11]. Why do cells secrete even essential metabolites so often? A simplistic answer would be that metabolites inevitably leak, regardless of whether the leakage inhibits cell growth. Although this is not unreasonable, especially for small molecules, and is consistent with some empirical observations [13-15], it is not clear why the cells have not evolved mechanisms to suppress the leakage. An alternative possibility is that there are some benefits for cells when they leak chemicals necessary for their growth. Is such advantageous leakage really possible for a class of intracellular metabolic

Published by the American Physical Society under the terms of the Creative Commons Attribution 4.0 International license. Further distribution of this work must maintain attribution to the author(s) and the published article's title, journal citation, and DOI. reactions, and if so, how is it possible and how general is it? Further, the coexistence of diverse cell types by utilizing leaked, useful chemicals will also be discussed.

To address these questions, we consider a dynamicalsystem model of a cell with simple metabolic reactions. Let us consider an isolated cell that contains $n$ kinds of chemical components (e.g., metabolites and enzymes) [16-18]. The cellular state is expressed by concentrations of the $n$ components, $\mathbf{x}={ }^{t}\left(x_{0}, x_{1}, \ldots, x_{n-1}\right)$. In the cell, chemical $i$ is synthesized and decomposed by a set of intracellular reactions with rate $F_{i}(\mathbf{x})$ and is exchanged with the environment at rate $f_{i}\left(\mathbf{x} ; D_{i}, x_{i}^{(\text {env) }}\right)$; if $f_{i}$ is positive, then chemical $i$ flows in from the environment, and if it is negative, chemical $i$ is leaked out. $D_{i}$ is a positive parameter characterizing the flow rate of each component $i$, and we call it the diffusion coefficient. The fixed non-negative parameter $x_{i}^{\text {(env) }}$ represents the $i$ th chemical's concentration in the environment: $x_{i}^{\text {(env) }}=S_{\text {env }}>0$ if chemical $i$ is an externally supplied nutrient, and $x_{i}^{\text {(env) }}=0$ otherwise. In the following we assume passive diffusion, $f_{i}\left(\mathbf{x} ; D_{i}, x_{i}^{(\text {env })}\right)=$ $D_{i}\left(x_{i}^{\text {(env) }}-x_{i}\right)$, but usage of active transport does not alter the results as much [19].

The time-dependent change in the $i$ th chemical's concentration $x_{i}$ is given by

$$
\dot{x}_{i}=F_{i}(\mathbf{x})+f_{i}\left(\mathbf{x} ; D_{i}, x_{i}^{(\text {env })}\right)-\mu\left(\mathbf{x} ; \mathbf{D}, \mathbf{x}^{(\text {env })}\right) x_{i},
$$

where $\mu\left(\mathbf{x} ; \mathbf{D}, \mathbf{x}^{(\mathrm{env})}\right)$ is the growth rate of the cellular volume and is determined by the synthesis of biomass (or membrane) component(s). Here, the third term represents the dilution of each chemical owing to the cellular volume increase [20]. We assume that a steady state (i.e., a stable fixed point) $\mathbf{x}=\mathbf{x}^{*}$ exists and is reached, where 

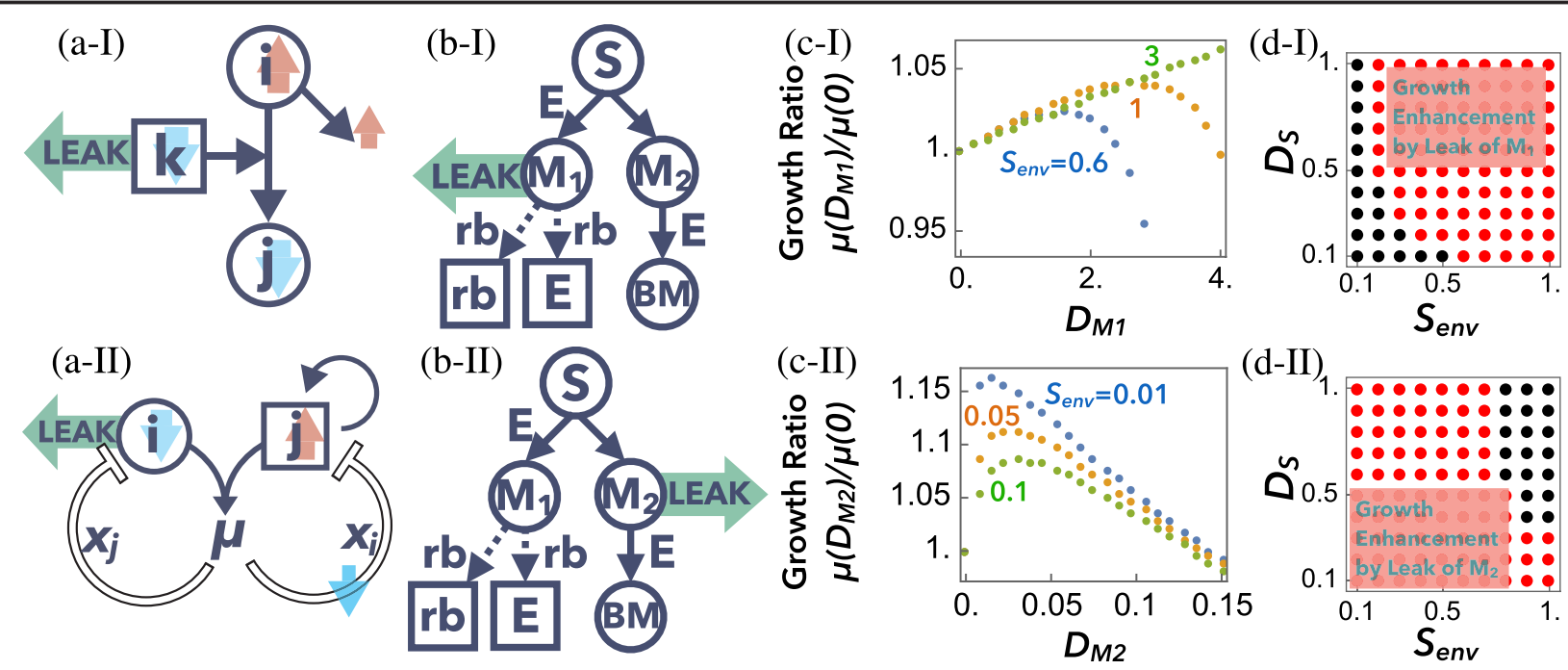

FIG. 1. The mechanisms underlying the leak advantage of an essential chemical: (I) metabolite $M_{1}$ or enzyme $E$ and (II) biomass precursor $M_{2}$. (see also Supplemental Material [21], Fig. S1). (a) Schematic illustration of (a-I) flux control mechanism and (a-II) growth-dilution mechanism. (b) Reaction network to illustrate the two mechanisms. Solid and dashed arrows show catalytic reactions and translations, respectively. In this example, leakage of metabolite $M_{1}$ or enzyme $E$ (b-I), or biomass precursor $M_{2}$ (b-II) can increase the growth rate $\mu(\mathbf{x}) \equiv k_{M_{2} \rightarrow \mathrm{BM}} x_{M_{2}} x_{E}$. (c) The relationship between the diffusion coefficient $D_{i}$ and growth ratio $\mu\left[\mathbf{x}\left(D_{i}\right)\right] / \mu\left[\mathbf{x}\left(D_{i}=0\right)\right]$ (with different $S_{\text {env }}$ and $D_{S}=1$ ). (c-I) The leaked chemical $i$ is metabolite $M_{1}$. Blue, orange, and green dots depict growth rates with $S_{\text {env }}=0.6,1,3$, respectively. (c-II) The leaked chemical $i$ is metabolite $M_{2}$. Blue, orange, and green dots depict growth rates with $S_{\text {env }}=0.01,0.05,0.1$. (d) Phase diagrams of the leak advantage with different parameters. According to numerical simulations from Eq. (1), infinitesimal leakage of chemical $M_{1}$ or $E$ (d-I) and $M_{2}$ (d-II) is advantageous at $\left(S_{\text {env }}, D_{S}\right)$ of red dots. The numerical simulations are conducted with rate constants $k_{S \rightarrow M_{1}}=1$ and $k_{M_{2} \rightarrow \mathrm{BM}}=0.01$ in (c-I) and (d-I), and with $k_{S \rightarrow M_{1}}=0.9$ and $k_{M_{2} \rightarrow \mathrm{BM}}=$ 0.25 in (c-II) and (d-II). The other rate constants are set at unity in both cases.

$\mathbf{x}^{*}$ satisfies $\mathbf{G}\left(\mathbf{x}^{*} ; \mathbf{D}, \mathbf{x}^{(\text {env })}\right)=\mathbf{0}$ with $G_{i}\left(\mathbf{x} ; \mathbf{D}, \mathbf{x}^{(\text {env })}\right) \equiv$ $F_{i}(\mathbf{x})+f_{i}\left(\mathbf{x} ; D_{i}, x_{i}^{(\mathrm{env})}\right)-\mu\left(\mathbf{x} ; \mathbf{D}, \mathbf{x}^{(\mathrm{env})}\right) x_{i}$.

Now consider a small change in diffusion coefficients: $\mathbf{D} \rightarrow \mathbf{D}+\delta \mathbf{D}$, where $\delta D_{i} \geq 0$ if chemical $i$ is not a nutrient and $\delta D_{i}=0$ otherwise. As long as chemical component $i$ is not externally supplied into the environment, an increase in the diffusion coefficient of the non-nutrient chemical $i$ leads to its additional leakage. Through this change, the steady state and growth rate also change as $\mathbf{x}^{*} \rightarrow \mathbf{x}^{*}+\delta \mathbf{x}$ and $\mu^{*} \equiv \mu\left(\mathbf{x}^{*} ; \mathbf{D}, \mathbf{x}^{(\text {env })}\right) \rightarrow \mu^{*}+\delta \mu$. We consider infinitesimal $\delta \mathbf{D}$ and analyze the values of $\delta \mathbf{x}$ and $\delta \mu$ by linearizing the equation $\dot{\mathbf{x}}=\mathbf{G}\left(\mathbf{x} ; \mathbf{D}, \mathbf{x}^{(\text {env })}\right)$. Then, by means of the Jacobi matrix $J=\partial \mathbf{G} /\left.\partial \mathbf{x}\right|_{\mathbf{x}=\mathbf{x}^{*}}, \delta \mu$ is derived as follows (see Supplemental Material [21], Sec. S1):

$$
\delta \mu=\left[\frac{\partial \mu}{\partial \mathbf{D}}+\frac{\partial \mu}{\partial \mathbf{x}} J^{-1}\left(\mathbf{x} \frac{\partial \mu}{\partial \mathbf{D}}-\frac{\partial \mathbf{f}}{\partial \mathbf{D}}\right)\right] \cdot \delta \mathbf{D}
$$

We now investigate how $\delta \mu$ can be made positive by leakage of a necessary chemical, whereas leakage of unnecessary chemicals is evidently advantageous, as seen in classical syntrophy [5-7]. To exclude the classical syntrophy, we assume that no chemicals directly inhibit biomass synthesis. When the leakage of useful chemicals promotes cell growth, we term them as "leak-advantage" chemicals.
First, note that leakage cannot be advantageous if intracellular chemical reactions consist of only one-body reactions like $i \rightarrow j$ (in general, $i \rightarrow j_{1}+\cdots+j_{m}$ ). Since the dynamics in this case are a linear function of $\mathbf{x}$, leakage cannot increase the reaction rate of biomass synthesis $\mu$, as analytically proven in Supplemental Material [21], Sec. S1.

In contrast, metabolic networks include multibody reactions, as most reactions are catalyzed by some other components like $i+k \rightarrow j+k$. Such nonlinearity in reactions changes the situation. Indeed, we numerically found that randomly generated catalytic reaction networks often exhibit the leak advantage, as will be described later. From these examples, we have extracted two basic mechanisms for the leak advantage: "flux control" and "growthdilution." The former mechanism is straightforward [see Fig. 1(a-I)]: catalytic reaction $i+k \rightarrow j+k$ is decelerated by leakage of catalyst $k$ (or a metabolite that produces $k$ ), which consequently decreases the abundance of product $j$ but increases that of substrate $i$. Thus, if $i$ also serves as a substrate or enzyme in another reaction, this reaction is accelerated. The latter mechanism is a result of the balance between biomass synthesis and growth-induced dilution, and is theoretically deeper and counterintuitive [see Fig. 1(a-II)]: increased growth enhances the dilution so that the concentration of each chemical is decreased. Thus, growth-induced dilution leads to negative feedback 
for the reactants of biomass synthesis ( $i$ and $j$ in the figure). As a result, leakage of $i$ relaxes this negative feedback for $j$ and thus can enhance cell growth $\mu$ if $j$ is involved in a autocatalytic module, as will be explained in depth later.

We here illustrate these two mechanisms through a simple example consisting of the minimal, common ingredients in cells: one substrate $(S)$, enzyme $(E)$, ribosome $(\mathrm{rb})$, and a few metabolites $\left(M_{1}\right.$ and $\left.M_{2}\right)$, as well as the biomass or biomembrane (BM) [Fig. 1(b)]. The example includes the following chemical reactions and translations (solid and dashed arrows, respectively) [22]:

$$
\begin{aligned}
S+E & \rightarrow M_{1}+E, \quad S \rightarrow M_{2}, \quad M_{2}+E \rightarrow \mathrm{BM}+E, \\
M_{1}+\mathrm{rb} & \rightarrow \mathrm{rb}+\mathrm{rb}, \quad M_{1}+\mathrm{rb} \rightarrow E+\mathrm{rb} .
\end{aligned}
$$

The evolution of the concentrations is given by

$$
\begin{aligned}
\dot{x}_{S}= & -k_{S \rightarrow M_{1}} x_{S} x_{E}-k_{S \rightarrow M_{2}} x_{S}+D_{S}\left(S_{\mathrm{env}}-x_{S}\right)-\mu x_{S} \\
\dot{x}_{M_{1}}= & k_{S \rightarrow M_{1}} x_{S} x_{E}-\left(k_{M_{1} \rightarrow \mathrm{rb}}+k_{M_{1} \rightarrow E}\right) x_{M_{1}} x_{\mathrm{rb}} \\
& -D_{M_{1}} x_{M_{1}}-\mu x_{M_{1}} \\
\dot{x}_{\mathrm{rb}}= & k_{M_{1} \rightarrow \mathrm{rb}} x_{M_{1}} x_{\mathrm{rb}}-\mu x_{\mathrm{rb}} \\
\dot{x}_{E}= & k_{M_{1} \rightarrow E} x_{M_{1}} x_{\mathrm{rb}}-D_{E} x_{E}-\mu x_{E} \\
\dot{x}_{M_{2}}= & k_{S \rightarrow M_{2}} x_{S}-k_{M_{2} \rightarrow \mathrm{BM}} x_{M_{2}} x_{E}-D_{M_{2}} x_{M_{2}}-\mu x_{M_{2}},
\end{aligned}
$$

where the growth rate is defined as the synthesis rate of biomass $\mathrm{BM}$ from its precursor $M_{2}$, so that $\mu(\mathbf{x}) \equiv$ $k_{M_{2} \rightarrow \mathrm{BM}} x_{M_{2}} x_{E}$. Here, leakage of ribosome $\mathrm{rb}$ is unlikely to occur and thus is not included.

The change in growth rate $\delta \mu$ due to leakage is obtained by numerically calculating the steady state $\mathbf{x}^{*}$ and Eq. (1). The two mechanisms mentioned above are demonstrated by the two cases: (I) leakage of metabolite $M_{1}$ (or enzyme $E$ ) [Fig. 1(b-I)] and (II) leakage of biomass precursor $M_{2}$ [Fig. 1(b-II)]. Although all chemicals are necessary for cell growth in this example, these leakages are advantageous over a wide range of parameters [Figs. 1(c) and 1(d)].

Flux control mechanism. - [Fig. 1(a-I)]: In case (I), the leakage of $M_{1}$ (i.e., an increase in $D_{M_{1}}$ ) decreases the flux from $M_{1}$ to $E$ and $x_{E}$. Accordingly, the reaction $S+E \rightarrow$ $M_{1}+E$ decelerates, which in turn raises $x_{S}$ and the flux $S \rightarrow M_{2}$. In this way, the growth rate $\mu(\mathbf{x})=k_{M_{2} \rightarrow \mathrm{BM}} x_{M_{2}} x_{E}$ increases [Figs. 1(c-I) and 1(d-I)]. Here, note that leakage of enzyme $E$ itself (instead of metabolite $M_{1}$ ) can also be advantageous, while leakage of enzymes is not so common.

Growth-dilution mechanism. - [Fig. 1(a-II)]: In case (II), the dilution term due to the volume growth matters. Here, we call the reactants for biomass synthesis (i.e., the precursor $M_{2}$ and enzyme $E$ for the biomass synthesis reaction in the example) "biomass producers." Then, generally, every biomass producer has negative feedback with itself: if the concentration of a biomass producer increases, then the rate of biomass synthesis and the dilution due to the volume growth increases, thereby suppressing its own concentration. For instance, the dilution term for $E$ in Fig. 1(b) is $-\mu x_{E}=-k_{M_{2} \rightarrow B M} x_{M_{2}} x_{E}^{2}$. Here, the magnitude of this negative feedback for a biomass producer (e.g., $E$ in the example) is weakened by reducing the concentration of the other biomass producer(s) (i.e., $x_{M_{2}}$ ). The concentration of $E$ thus increases by leaking $M_{2}$. Because of the nonlinear autocatalytic processes for $E$ through $\mathrm{rb}$ and $M_{1}$, this increase can surpass the loss of the leaked biomass producer $M_{2}$; then the biomass synthesis rate, $k_{M_{2} \rightarrow \mathrm{BM}} x_{M_{2}} x_{E}$, is enhanced [Figs. 1(c-II) and 1(d-II)]. The leakage of a biomass producer, thus, can be advantageous.

One can check this analytically as follows. From $\dot{x}_{\mathrm{rb}}=0$ and $\dot{x}_{E}=0, x_{\mathrm{rb}}=c x_{E}$ and $k_{M_{1} \rightarrow \mathrm{rb}} x_{M_{1}}=\mu$ hold in the steady state, with $c \equiv k_{M_{1} \rightarrow \mathrm{rb}} / k_{M_{1} \rightarrow E}$; here we assume $D_{E}=D_{M_{1}}=0$ because only the leakage of $M_{2}$ is considered in this case (II), whereas even if they are positive, their values do not change the conclusion. From $\dot{x}_{M_{1}}=0$, it follows $x_{M_{1}}=k_{S \rightarrow M_{1}} x_{S} x_{E} /\left(\mu+c^{\prime} x_{E}\right)=\mu / k_{M_{1} \rightarrow \mathrm{rb}}$, with $\quad c^{\prime} \equiv c\left(k_{M_{1} \rightarrow \mathrm{rb}}+k_{M_{1} \rightarrow E}\right)$. Substituting $\quad \mu(\mathbf{x})=$ $k_{M_{2} \rightarrow \mathrm{BM}} x_{M_{2}} x_{E}$ in the left-hand side of this equation, we gain $k_{M_{1} \rightarrow \mathrm{rb}} k_{S \rightarrow M_{1}} x_{s} /\left(k_{M_{2} \rightarrow \mathrm{BM}} x_{M_{2}}+c^{\prime}\right)=\mu$. The decrease in $x_{M_{2}}$ by leaking $M_{2}$ decreases the denominator. Hence, the steady growth rate $\mu^{*}$ increases if the change in $x_{S}$ is sufficiently smaller than the decrease in the denominator. This condition is satisfied when the reaction $S \rightarrow M_{2}$ is much faster than the reaction $S+E \rightarrow M_{1}+E$, or when $k_{M_{2} \rightarrow \mathrm{BM}}$ is large enough (Supplemental Material [21], Fig. S1). Since the balance between biomass synthesis and growth-induced dilution determines the cellular steady state $\mathbf{x}^{*}$, a self-consistent equation can also be used to calculate $\mu^{*}$ and $\delta \mu$ (see Supplemental Material [21], Sec. S2 and Fig. S2 for details).

The above simple illustration suggests that the existence of autocatalytic module(s) (i.e., a positive feedback process to enhance its own reactions and concentrations) is essential for both mechanisms, as discussed below. One can see this property in Fig. 1(b): the synthesis of $E$ involves nonlinear autocatalytic processes as the precursor synthesis $M_{1}$ is catalyzed by $E$ and the synthesis of $E$ is catalyzed by $\mathrm{rb}$ that is synthesized from $M_{1}$.

For the flux control mechanism, positive feedback for the autocatalytic module may work excessively under certain conditions. In case (I), when $S_{\text {env }}$ or the rate constant for the reaction into the autocatalytic module, $k_{S \rightarrow M_{1}}$, is large or when the rate constant $k_{M_{2} \rightarrow \mathrm{BM}}$ is small, this excessive production occurs and the leak advantage for $M_{1}$ or $E$ appears [see Fig. 1(d-I)]. Of note, the leakage of $M_{1}$ counterintuitively increases $x_{M_{1}}$ when it enhances cell growth; this is a consequence of nonlinear autocatalytic process and growth-dilution balance, as it is simply proven from the steady condition $\dot{x}_{\mathrm{rb}}=0$ leading to $k_{M_{1} \rightarrow \mathrm{rb}} x_{M_{1}}=\mu$. 
With regard to the growth-dilution mechanism, a nonlinear autocatalytic process for $E$ is necessary to increase the growth rate more than the direct decrease by leaking $M_{2}$. Note that this mechanism works even when the nutrient supply is scarce [23], as it is based on negative feedback via the growth-induced dilution. In Figs. 1(c-II) and (d-II), the smaller the nutrient supply, the broader the parameter region for the leak advantage for $M_{2}$ and the larger the growth change $\delta \mu / \mu$. As long as the rate constant for biomass synthesis $k_{M_{2} \rightarrow \mathrm{BM}}$ is large or the rate constant $k_{S \rightarrow M_{1}}$ is small, the negative feedback due to the volumegrowth dilution is relatively significant and thus this mechanism works.

Next, to examine if, and how commonly, leakage of a useful chemical can promote cell growth, we randomly generated thousands of chemical reaction networks consisting of metabolites and enzymes. Then, in the environmental condition fixed as $S_{\text {env }}=0.1$ and $D_{S}=1$, we checked whether the growth with each network is enhanced by increasing the diffusion coefficient, $D_{i}$, of each non-nutrient component $i$.

We considered reaction networks including only catalytic reactions $i+k \rightarrow j+k$ with a catalyst $k$ and the equal rate constants (set at unity) as the simplest multibody reactions. Out of $n$ chemical components in each network, $N_{\text {enzyme }}$ chemicals are "enzymes," which can be the catalyst $k$ or product $j$ of each reaction, and a single nutrient and the rest of the chemicals ("metabolites") can be the substrate $i$ or product $j$ of each reaction (see Supplemental Material [21], Sec. S3 for details and examples of randomly generated networks). To explicitly include the cost of leakage, we here assume that all chemicals contribute equally to the cell volume: the growth rate is then defined as the gain rate of total components, $\mu\left(\mathbf{x} ; \mathbf{D}, \mathbf{x}^{(\mathrm{env})}\right) \equiv$ $\sum_{i} f_{i}\left(\mathbf{x} ; D_{i}, x_{i}^{\text {(env) }}\right)$. Then, the first term of Eq. (1) is always negative; i.e., the leakage of non-nutrient chemicals, by itself, always decreases the cell volume. The leak advantage can, however, generally appear even in this severest case because the second term of Eq. (1) can be positive and surpass the first term [24].

Figure 2 depicts the proportion of networks having a leak-advantage chemical (either an enzyme or metabolite). Here, the number of networks with leak-advantage metabolite(s), enzyme(s), or chemical(s) in total are plotted against path density $\rho$ that is defined as the number of all the reactions $(\rho n)$ divided by the number of chemicals $n$. Remarkably, the proportion of leak-advantage networks is greater than $50 \%$ at $\rho=1.5$ to 3.0 in the case of $n=20$ [Fig. 2(a)], and Fig. 2(b) presents that this proportion and the average numbers of leak-advantage chemicals increase with $n$. That is, the presence of leak-advantage metabolites and enzymes is a generic property of complicated catalytic reaction networks as is the case for metabolic networks in actual cells.
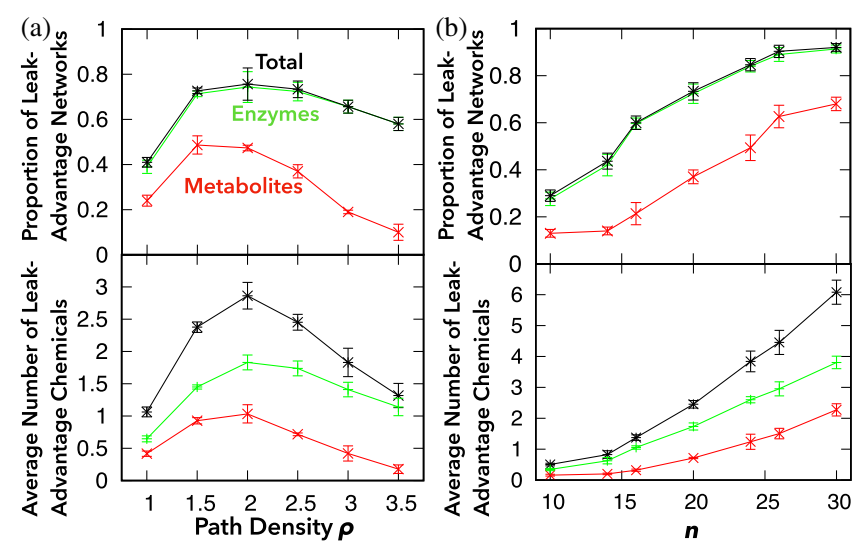

FIG. 2. Statistics of the leak advantage for randomly generated networks. Three hundred networks were randomly generated for each set of parameters with $N_{\text {enzyme }}=n / 2-1$. (a) Path density dependence of the proportion of leak-advantage networks (top) and of the average number of leak-advantage chemicals (bottom). The number of chemicals $n$ is set to 20. (b) The dependence on $n$ of the proportion of leak-advantage networks (top) and of the average number of leak-advantage chemicals (bottom) with $\rho=2.5$. Here, red, green, and black lines show the values for leakage of metabolites, enzymes, and chemicals in total, respectively, and the error bars indicate 1 standard error.

Here, as $n$ increases, there can be more autocatalytic modules of chemicals; this seems to be the reason why leak-advantage networks and chemicals are more common with larger $n$ [Fig. 2(b)]. The peak in the commonness of leak advantage at finite $\rho$ in Fig. 2(a) can also be understood: if the path density $\rho$ is too low, each chemical is rarely involved in autocatalytic modules or in multiple reactions. In contrast, if the path density $\rho$ is too high, all of the chemical reactions are extremely tangled, and the leakage of a chemical will reduce the flux of every reaction on average, and increasing specific reactions for biomass synthesis will be harder.

To summarize, we have analytically and numerically investigated dynamical-system models of an isolated cell with simple metabolic reactions, showing that leakage of essential chemicals (metabolites or enzymes) can enhance cell growth even under nutrient limitation. We also found that such advantageous leakage becomes increasingly common as the number of components in metabolic reaction networks increases. One mechanism for this is flux control to enhance the reactions contributing to cell growth. In the other general mechanism via global dilution due to cellular volume growth, leakage of essential reactants for biomass synthesis can modify the negative feedback due to their dilution and thereby enhance biomass synthesis. For both mechanisms, autocatalytic processes and growth-dilution balance are important. We confirmed that these general mechanisms actually work even in randomly generated catalytic networks (see Supplemental Material [21], Sec. S3 and Fig. S7). 
The leak advantage discussed above can be a plausible evolutionary first step for the diversity and symbiosis in a microbial ecosystem. The origins of microbial ecosystems of diverse cell types have been extensively studied [2-4,25-27]. In particular, Morris et al. stressed the importance of chemical leakage by proposing the black queen hypothesis, a theory on the evolution of metabolic dependency based on gene loss [13,28]. These studies, however, basically assume that the properties of a permeable membrane inevitably induce metabolite secretion, leading to parasitism or free riding of other cells. In this respect, our results offer a fresh look: some cells may secrete essential chemicals simply because this leakage is beneficial for them. As cells that leak essential metabolites are crowded, such metabolites accumulate in the environment, preventing further leakage. The presence of another cell type that consumes the leaked metabolites for its growth then may facilitate further leakage and benefit the "leaker" cells, whereas the useful metabolites secreted by the leaker cells support the growth of the "consumer" cells. As a result, their coexistence enhances the growth of both, providing a different form of division of labor than usual $[29,30]$. Indeed, numerical simulations with interacting cells, rather than isolation conditions, demonstrate that the leak advantage allows for the coexistence of diverse cells, following this novel form of symbiosis, as will be published elsewhere [31].

The authors would like to thank Chikara Furusawa for useful comments. This research was partially supported by a Grant-in-Aid for Scientific Research (S) (15H05746) and Grant-in-Aid for Scientific Research on Innovative Areas (17H06386) from the Ministry of Education, Culture, Sports, Science and Technology (MEXT) of Japan.

*saito@ubi.s.u-tokyo.ac.jp

†kaneko@complex.c.u-tokyo.ac.jp

[1] R. F. Rosenzweig, R. R. Sharp, D. S. Treves, and J. Adams, Microbial evolution in a simple unstructured environment: Genetic differentiation in Escherichia coli, Genetics 137, 903 (1994).

[2] J. E. Goldford, N. Lu, D. Bajić, S. Estrela, M. Tikhonov, A. Sanchez-Gorostiaga, D. Segrè, P. Mehta, and A. Sanchez, Emergent simplicity in microbial community assembly, Science 361, 469 (2018).

[3] A. Goyal and S. Maslov, Diversity, Stability, and Reproducibility in Stochastically Assembled Microbial Ecosystems, Phys. Rev. Lett. 120, 158102 (2018).

[4] A. Zelezniak, S. Andrejev, O. Ponomarova, D. R. Mende, P. Bork, and K. R. Patil, Metabolic dependencies drive species co-occurrence in diverse microbial communities, Proc. Natl. Acad. Sci. U.S.A. 112, 6449 (2015).

[5] B. E. L. Morris, R. Henneberger, H. Huber, and C. Moissl-Eichinger, Microbial syntrophy: Interaction for the common good, FEMS Microbiol. Rev. 37, 384 (2013).
[6] M. Cavaliere, S. Feng, O.S. Soyer, and J. I. Jiménez, Cooperation in microbial communities and their biotechnological applications, Environ. Microbiol. 19, 2949 (2017).

[7] B. Schink, Energetics of syntrophic cooperation in methanogenic degradation, Microbiol. Mol. Biol. Rev. 61, 262 (1997).

[8] T. Pfeiffer and S. Bonhoeffer, Evolution of crossfeeding in microbial populations, Am. Nat. 163, E126 (2004).

[9] T. G. Wilkinson, H. H. Topiwala, and G. Hamer, Interactions in a mixed bacterial population growing on methane in continuous culture, Biotechnol. Bioeng. 16, 41 (1974).

[10] E. E. Lilja and D. R. Johnson, Segregating metabolic processes into different microbial cells accelerates the consumption of inhibitory substrates, ISME J. 10, 1568 (2016).

[11] N. Paczia, A. Nilgen, T. Lehmann, J. Gätgens, W. Wiechert, and S. Noack, Extensive exometabolome analysis reveals extended overflow metabolism in various microorganisms, Microb. Cell Fact. 11, 122 (2012).

[12] L. P. Silva and T. R. Northen, Exometabolomics and MSI: Deconstructing how cells interact to transform their small molecule environment, Curr. Opin. Biotechnol. 34, 209 (2015).

[13] J. J. Morris, Black queen evolution: The role of leakiness in structuring microbial communities, Trends Genet. 31, 475 (2015).

[14] J. Gore, H. Youk, and A. van Oudenaarden, Snowdrift game dynamics and facultative cheating in yeast, Nature (London) 459, 253 (2009).

[15] Z. Wang and N. Goldenfeld, Theory of cooperation in a micro-organismal snowdrift game, Phys. Rev. E 84, 020902(R) (2011).

[16] K. Kaneko and T. Yomo, Cell division, differentiation and dynamic clustering, Physica (Amsterdam) 75D, 89 (1994).

[17] C. Furusawa and K. Kaneko, Zipfs Law in Gene Expression, Phys. Rev. Lett. 90, 088102 (2003).

[18] C. Furusawa and K. Kaneko, Adaptation to Optimal Cell Growth Through Self-Organized Criticality, Phys. Rev. Lett. 108, 208103 (2012).

[19] In the case of active transport, the uptake and secretion rates are formulated as $f_{i}\left(\mathbf{x} ; D_{i}, x_{i}^{(\text {env })}\right)=D_{i} x_{i}^{(\text {env })}$ and $=-D_{i} x_{i}$, respectively [18].

[20] D. J. Kiviet, P. Nghe, N. Walker, S. Boulineau, V. Sunderlikova, and S. J. Tans, Stochasticity of metabolism and growth at the single-cell level, Nature (London) 514, 376 (2014).

[21] See Supplemental Material at http://link.aps.org/ supplemental/10.1103/PhysRevLett.124.048101 for proofs of analytical results, the self-consistent equation approach, and additional data on examples of a leak advantage.

[22] The reaction system in Fig. 1(b) is presented to exemplify the two general mechanisms. Each "chemical variable" (and reaction) can also be interpreted as a cluster of molecules classified into categories, instead of a single specific molecule or elementary biochemical reaction.

[23] Note that, for some networks, the flux control mechanism can also work when the nutrient supply is scarce; besides, a leak advantage is also possible even in a chain reaction system without a chemical working as substrates for 
multiple reaction like the example in Fig. 1(b) (see Supplemental Material [21], Sec. S2).

[24] The results are qualitatively reproduced for a model that includes the ribosomal chemical and translation processes, and for models in which the growth rate is determined by the synthesis of only a single biomass chemical (see Supplemental Material [21], Sec. S3). It also suggests the commonness of a leak advantage.

[25] A. Kashiwagi, W. Noumachi, M. Katsuno, M. T. Alam, I. Urabe, and T. Yomo, Plasticity of fitness and diversification process during an experimental molecular evolution, J. Mol. Evol. 52, 502 (2001).

[26] T. Großkopf, J. Consuegra, J. Gaffé, J. C. Willison, R. E. Lenski, O.S. Soyer, and D. Schneider, Metabolic modelling in a dynamic evolutionary framework predicts adaptive diversification of bacteria in a long- term evolution experiment, BMC Evol. Biol. 16, 163 (2016).

[27] A. R. Zomorrodi and D. Segré, Genome-driven evolutionary game theory helps understand the rise of metabolic interdependencies in microbial communities, Nat. Commun. 8, 1563 (2017).

[28] J. J. Morris, R. E. Lenski, and E. R. Zinser, The black queen hypothesis: Evolution of dependencies through adaptative gene loss, MBio 3, e00036 (2012).

[29] J. F. Yamagishi, N. Saito, and K. Kaneko, Symbiotic cell differentiation and cooperative growth in multicellular aggregates, PLoS Comput. Biol. 12, e1005042 (2016).

[30] E. Flores and A. Herrero, Compartmentalized function through cell differentiation in filamentous cyanobacteria, Nat. Rev. Microbiol. 8, 39 (2010).

[31] J. F. Yamagishi, N. Saito, and K. Kaneko (to be published). 